\title{
Adaptive optics ophthalmologic systems using dual deformable mirrors
}

S.M. Jones, S. Olivier, D. Chen, S. Sadda, S. Joeres, R.J. Zawadzki, J. S. Werner, D.T. Miller

February 6, 2007

Photonics West - SPIE San Jose, CA, United States January 20, 2007 through January 25, 2007 
This document was prepared as an account of work sponsored by an agency of the United States Government. Neither the United States Government nor the University of California nor any of their employees, makes any warranty, express or implied, or assumes any legal liability or responsibility for the accuracy, completeness, or usefulness of any information, apparatus, product, or process disclosed, or represents that its use would not infringe privately owned rights. Reference herein to any specific commercial product, process, or service by trade name, trademark, manufacturer, or otherwise, does not necessarily constitute or imply its endorsement, recommendation, or favoring by the United States Government or the University of California. The views and opinions of authors expressed herein do not necessarily state or reflect those of the United States Government or the University of California, and shall not be used for advertising or product endorsement purposes. 


\title{
Adaptive Optics Ophthalmologic Systems using dual deformable mirrors
}

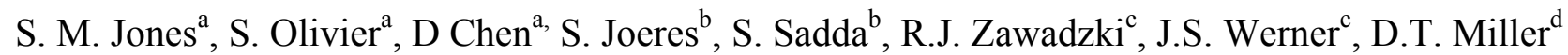 \\ ${ }^{a}$ Lawrence Livermore National Laboratory, 6000 East Avenue, Livermore, CA. 94539 \\ ${ }^{\mathrm{b}}$ Doheny Eye Institute, 1450 San Pablo Street, Los Angeles, CA. 90033 \\ 'Univ. of Calif./Davis Med Center, 4860 Y Street, Suite 2400, Sacramento, CA. 95817 \\ ${ }^{\mathrm{d}}$ Indiana University, 800 E. Atwater Ave., School of Opt., Bloomington, IN 47405
}

\begin{abstract}
Adaptive Optics (AO) have been increasingly combined with a variety of ophthalmic instruments over the last decade to provide cellular-level, in-vivo images of the eye. The use of MEMS deformable mirrors in these instruments has recently been demonstrated to reduce system size and cost while improving performance. However, currently available MEMS mirrors lack the required range of motion for correcting large ocular aberrations, such as defocus and astigmatism.

In order to address this problem, we have developed an AO system architecture that uses two deformable mirrors, in a woofer / tweeter arrangement, with a bimorph mirror as the woofer and a MEMS mirror as the tweeter. This setup provides several advantages, including extended aberration correction range, due to the large stroke of the bimorph mirror, high order aberration correction using the MEMS mirror, and additionally, the ability to 'focus' through the retina.

This AO system architecture is currently being used in four instruments, including an Optical Coherence Tomography (OCT) system and a retinal flood-illuminated imaging system at the UC Davis Medical Center, a Scanning Laser Ophthalmoscope (SLO) at the Doheny Eye Institute, and an OCT system at Indiana University. The design, operation and evaluation of this type of AO system architecture will be presented.
\end{abstract}

Keywords: Adaptive Optics, AO, dual deformable mirrors, retinal focus

\section{INTRODUCTION}

The resolution of scanning laser ophthalmoscopes (SLO) [1-2] and Optical Coherence Tomography (OCT) [15-16] instruments has continued to push the limits of optical system quality, light collimation and light collection efficiency and techniques. SLO [3-6] and OCT [17-19] instruments, using real time Adaptive Optics correction (AO) to remove both the ocular and system aberrations, have increased the image quality of the science imaging system by: 1) Improving (reducing) the spot size of the incident science illumination beam and AO beacon at the retina and 2) Eliminating ocular and system aberrations of the light returned from the eye, allowing efficient transmission and recording of the science beam. Without the real time Adaptive Optics correction, system effectiveness would be reduced by the degraded PSF at the confocal aperture in the SLO and at the fiber receiver in the sample arm of the OCT instruments.

Improvements in DM technology have allowed cost effective and practical AO ophthalmologic systems to be placed in clinical sites. As DM technologies have progressed, so have their application and incorporation into more refined instruments. Ophthalmologic instruments have been built, with varying success, using different deformable mirror types, including those with piezoelectric actuators, liquid crystal spatial light modulators, and micro-electro-mechanical-system (MEMS) mirrors [7-10]. 
As AO ophthalmologic instruments are moved into the clinical environment, clinician's ease-of-use becomes a priority. Studies have shown that the ocular aberrations of the general populous are composed of high amplitude, low-order, aberrations and lower amplitude, high-order, aberrations [11-12]. Current DM technology does not provide both the necessary high stroke and high order correction that are required for this combination of aberrations in the general population. Past systems have used several techniques to get around this limitation. These techniques include carefully selecting trial lenses or using other corrective techniques [10], to correct the lower order aberrations, and/or the subject pool is limited to individuals that fall within the prescribed limits of the system. Although these methods have and will continue to be used, they are cumbersome, time consuming, and limiting in scope.

Today, we have several mirrors $[9,14]$ that provide an interesting alternative to using a single deformable mirror and its associated limitations. To address the situation, we investigated the use of two deformable mirrors in the AO ophthalmologic instruments. The first of the two deformable mirrors, the "woofer DM", provides low-order aberrations with relatively large amplitudes and a second DM, the "tweeter DM", is used to correct high-order aberrations with lower amplitudes. This combination of the "woofer - tweeter deformable mirrors" provides a significant improvement in the clinical user interface and makes significant strides in correcting the real-time ocular aberrations, necessary to achieve diffraction-limited in-vivo retinal images.

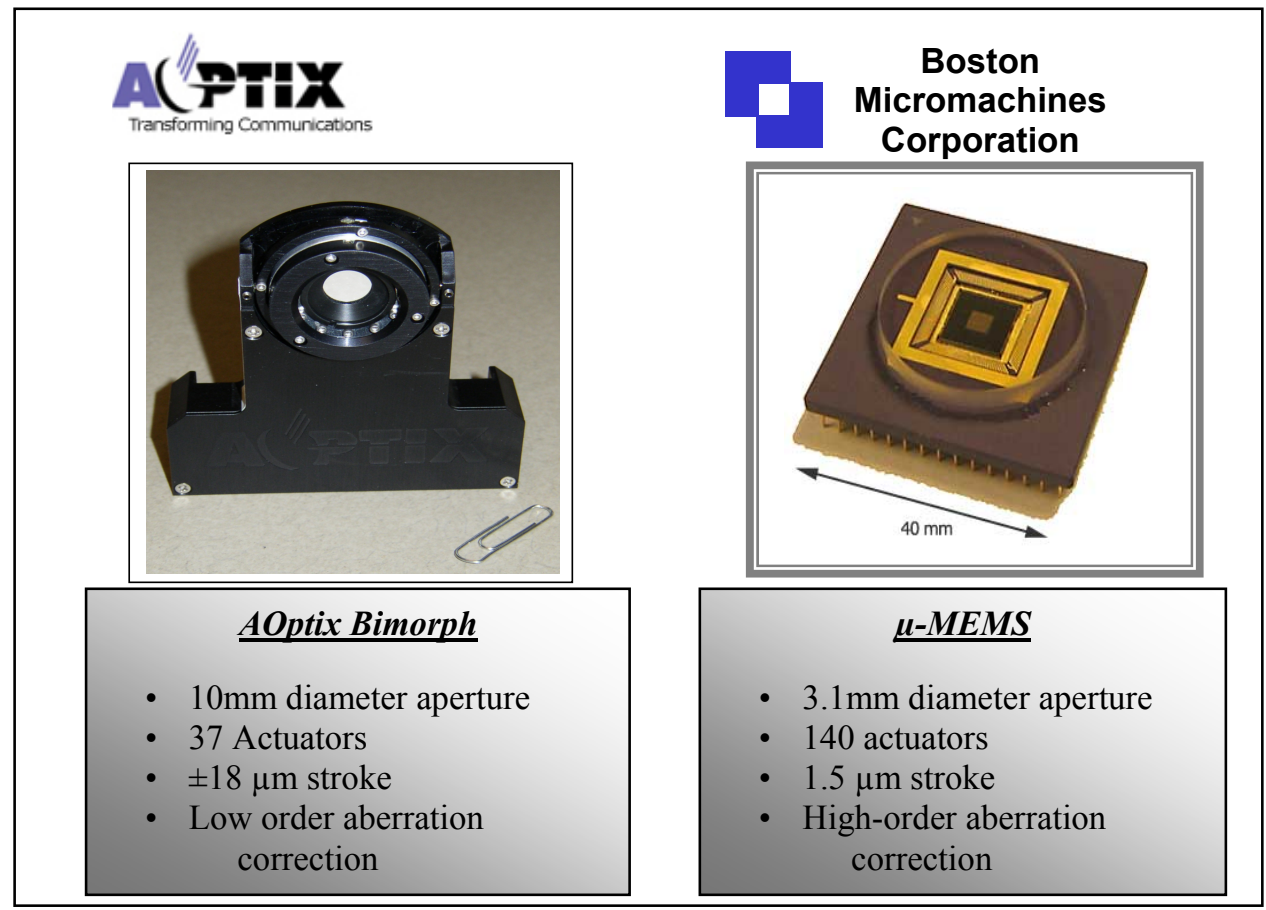

Figure 1 - Dual Deformable Mirrors: The AOptix Bimorph, "woofer", and the BMC $\mu$-MEMS, tweeter, deformable mirrors are used in the Dual DM Ophthalmic Instruments.

This paper will discuss the implementation and operation of the dual deformable mirror Adaptive Optics system that we have implemented in a semi-portable scanning laser ophthalmoscope and a brief overview of the performance of the AO system on human subjects. 


\section{METHODOLOGY}

\section{AO-SLO Deformable Mirror Technology}

Dual deformable mirror (DM) ophthalmologic instruments have now been designed with two mirrors correcting in the optical path. One mirror is a "woofer" DM, which is a 37 actuator, Bimorph deformable mirror made by the AOptix Corporation [16]. The second mirror is a "tweeter" DM, which is the 140 actuator MEMS deformable mirror made by Boston Micromachines Corporation [9]. See Figure \#1.

Table 1 - Mirror Characteristics in Current Three systems: The mirror surface coating is selected based on wavelength used for science images and overall system use.

\begin{tabular}{|c|c|c|c|}
\hline System Type & Location & $\underline{\mu-M S}$ & AOptix Bimorph \\
\hline AO-OCT & UC Davis Medical Center & $\begin{array}{l}\text { - } 12 \times 12 \text { Gold actuators } \\
\text { - } 300 \text { um pitch }\end{array}$ & $\begin{array}{l}\text { - Gold Surface } \\
\text { - } 10 \mathrm{~mm} \text { aperture }\end{array}$ \\
\hline AO-OCT & Univ. of Indiana & $\begin{array}{l}\text { - } 12 \times 12 \text { Gold actuators } \\
\text { - } 300 \text { um pitch }\end{array}$ & $\begin{array}{l}\text { - Gold Surface } \\
\text { - } 10 \mathrm{~mm} \text { aperture }\end{array}$ \\
\hline AO-SLO & $\begin{array}{l}\text { Doheny Eye Institute (USC } \\
\text { / Univ. of Rochester) }\end{array}$ & $\begin{array}{l}\text { - 12x12 Aluminum. actuators } \\
\text { - 300um pitch }\end{array}$ & $\begin{array}{l}\text { - Aluminum Surface } \\
\text { - } 10 \mathrm{~mm} \text { aperture }\end{array}$ \\
\hline
\end{tabular}


Table 2 - Common Attributes between the AO Systems: Although there are significant differences in the optical systems, the AO subsystem is designed to be modular, reusable, and functionality similar between systems.

\begin{tabular}{|c|c|}
\hline$\underline{\text { AO Attribute }}$ & Associated Parameters \\
\hline $\begin{array}{l}\text { WFS Camera [Dalsa 1M60 } \\
\text { Pantera] }\end{array}$ & $\begin{array}{l}\text { - }>30 \mathrm{~Hz} \text { ( Networked UI ) Typically run at } 20 \mathrm{~Hz} \text { due to system light limits } \\
\text { - Camera array is } 512 \times 512 \text { pixels }-4095 \mathrm{ADU} \\
\text { - Pixels (binned) are } 24 \mathrm{um} \text { by } 24 \mathrm{um}\end{array}$ \\
\hline Lenslets [AOA \#0500-30-S-A] & $\begin{array}{l}\text { - Lenslet array is } 20 \times 20 \mathrm{sq}, 500 \mathrm{um} \text { pitch, fl } \sim=30 \mathrm{~mm} \\
\text { - } 19 \times 19 \text { WFS camera pixels / centroid } \\
\text { - Circular lenslet mask defined - Using approx. } 280 \text { lenslets } \\
\text { - Corresponds to } 6-7 \mathrm{~mm} \text { at eye (system dependent) }\end{array}$ \\
\hline Centroid Calculations & $\begin{array}{l}\text { - Center of mass ( correlation calculation in-process) } \\
\text { - Threshold set to establish the noise floor }\end{array}$ \\
\hline $\begin{array}{l}\text { Light Source: [Superlum SLD- } \\
\text { 371-HP2 \&SLD-261-HP1] }\end{array}$ & $\begin{array}{l}\text { - Typically used at } \sim 830 \mathrm{~nm} \text { SLD with } 10-15 \mathrm{~nm} \text { bandwidth } \\
\text { - AO-SLO system allows } 680 \mathrm{~nm} \text { (blood vessel) or } 790 \text { (ICG) } \mathrm{nm} \\
\text { - A common light source (beam) is used for both AO Beacon and imaging }\end{array}$ \\
\hline AO Control System Software & $\begin{array}{l}\text { - Currently based on object based, embedded “AO-Control" software } \\
\text { - Configurable } \\
\text { - Networked }\end{array}$ \\
\hline Operating System / GUI & $\begin{array}{l}\text { - Windows XP } \\
\text { - Networked User interface } \\
\text { - } \text { Currently JAVA Based GUI ( Moving to LabWindows CVI - "C" / Linux) }\end{array}$ \\
\hline
\end{tabular}

There are currently three ophthalmological instruments that are successfully using the dual deformable mirror technology. Table \#1 lists the current instrument's configuration specific details. The selection of mirror coatings is based upon the anticipated wavelength requirements of the systems and on the available coating for the DMs [13]. Gold is an excellent reflector for wavelengths greater than $800 \mathrm{~nm}$. This works well in the OCT systems that typically use the Super Luminescent Diodes centered at $830 \mathrm{~nm}$ to $850 \mathrm{~nm}$. The SLO system, at the Doheny Eye Institute, will use aluminum coatings for wavelengths as short as $500 \mathrm{~nm}$.

The AO systems seen listed in Table \#1 are applied to very different types of optical systems. This includes two optically distinct AO-OCT systems and an AO-SLO system. In all cases, the AO subsystem is designed and maintained as a modular subsystem. The common attributes of the AO subsystems are shown in Table \#2.

The block diagram of a typical AO system is shown in Figure \#2. The AO system block diagram is shown distributed into five subsections: 1) Wavefront Sensor, 2) Centroid Calculations, 3) Difference matrix control, 4) Correction Wavefront Calculator, and 5) Deformable mirror and associated drivers. Each subsection, shown and enumerated above, incorporates multiple processes to complete its task. The associated text box lists the major processes for each subsection. 


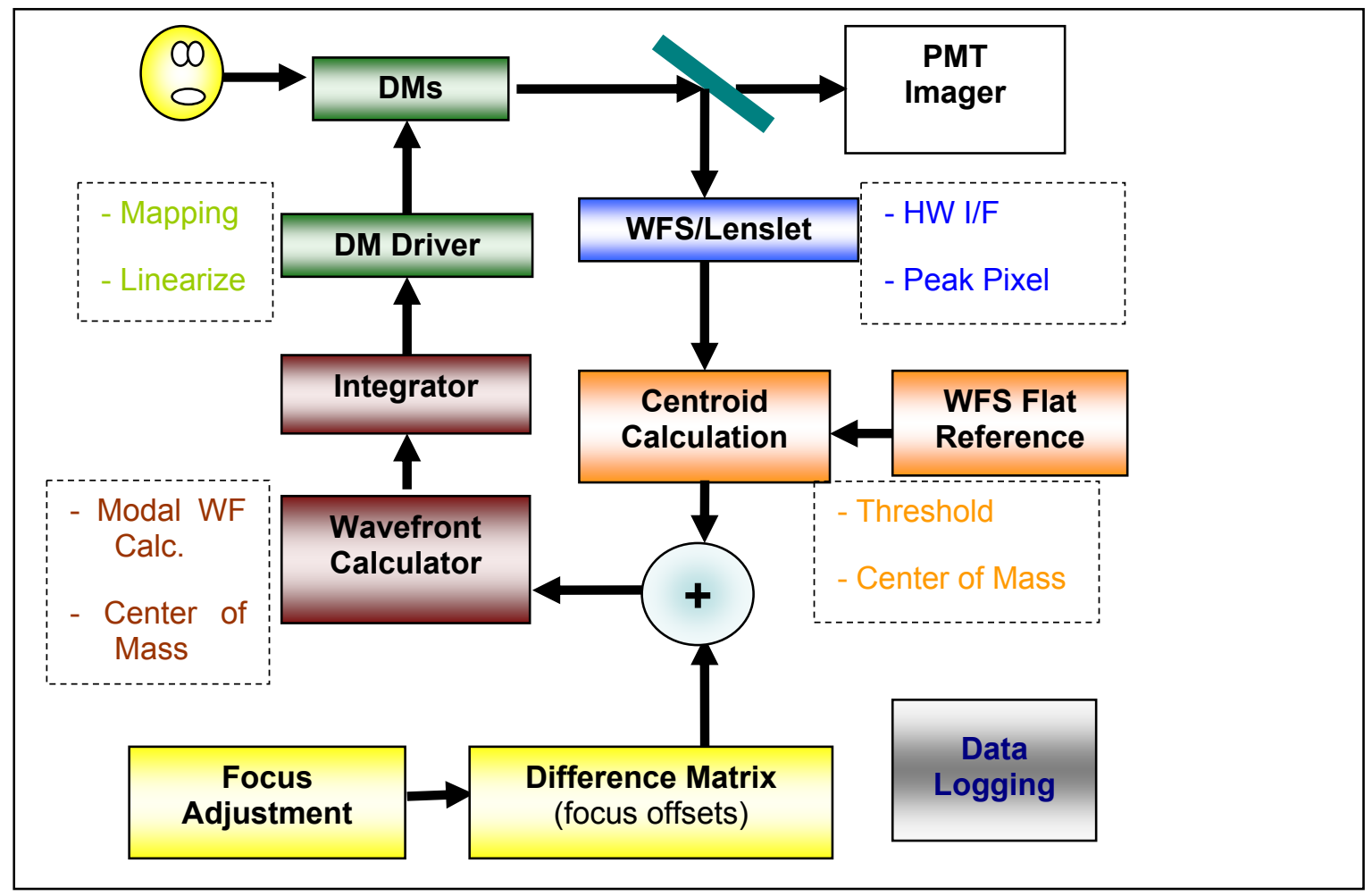

Figure 2 - Block diagram of the AO system: The block diagram shows the configuration of the AO system that is used in the Ophthalmological systems described here.

\section{Optical System support for Adaptive Optics}

The optical system required to implement an AO ophthalmologic instrument is significantly more complex than an equivalent non-AO system. A primary requirement on the optics design is that all moving surfaces, including the DMs and the scanning mirrors, must be located at conjugate pupil planes. The pupil of the eye is re-imaged at each of these points and it is necessary for this constraint to be met, to allow the error at the pupil of the eye to be measured and a correction to be properly applied by the DMs.

If the pupil conjugate planes are not properly aligned, the input and output beams will translate across the pupil of the eye. This motion occurs at a rate that is higher than the AO system can correct. Conversely, if the light passing through the pupil remains at a stationary position on the pupil, the wavefront error is correctly measured and minimized at the WFS and DMs respectively. Figure \#3 shows the current optical layout for the semi-portable system that is currently used at Doheny. 


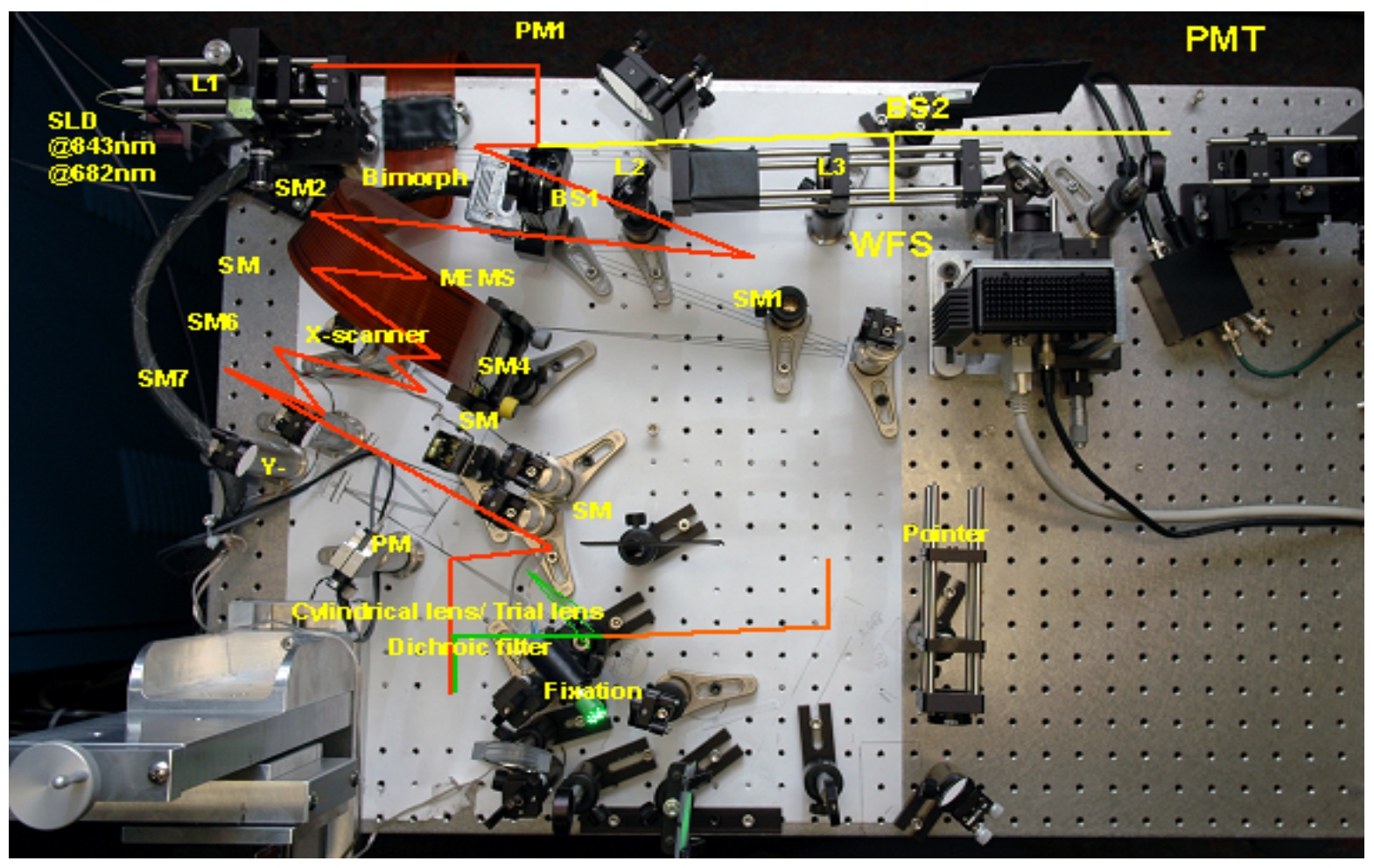

Figure 3 - Top view of the Optics for the Dual DM system: This is the AO-SLO system showing the AOptix Bimorph, "woofer" DM, and the BMC $\mu$-MEMS, "tweeter" DM. Legend: SLD: Super Luminescent Diode; L: lens; BS: Beam splitter; PM: Plane Mirror: SM: Spherical Mirror

The ocular aberration of the eye affects the incoming science/imaging beam, which is also the Adaptive Optics beacon, in the same manner as it does the reflected beam from the spot at the retina. By using the optical system in a "two-pass" mode, the incoming science beam is preconditioned so that the error seen at the eye is cancelled out and the spot at the retina is as close to diffraction limited as possible. This, however, also imposes tighter constraints on the system alignment because all incoming and outgoing apertures must be aligned within about $1 / 10$ of the actuator size of the MEMS deformable mirror.

\section{Overview of the Dual DM Current Operation}

Figure \#4 is a schematic diagram of the dual DM operation. The operating mode of the AO system currently requires an operator to manually switch between the DMs using the user interface.

This functionality will be enhanced to automate the time slicing during normal operation. As further experience is gained with the system and more data are collected from human subjects, this operation will be further enhanced to respond to changes in the wavefront rms and system modes, correctly selecting the appropriate mirror to optimize the operations of the DMs. 


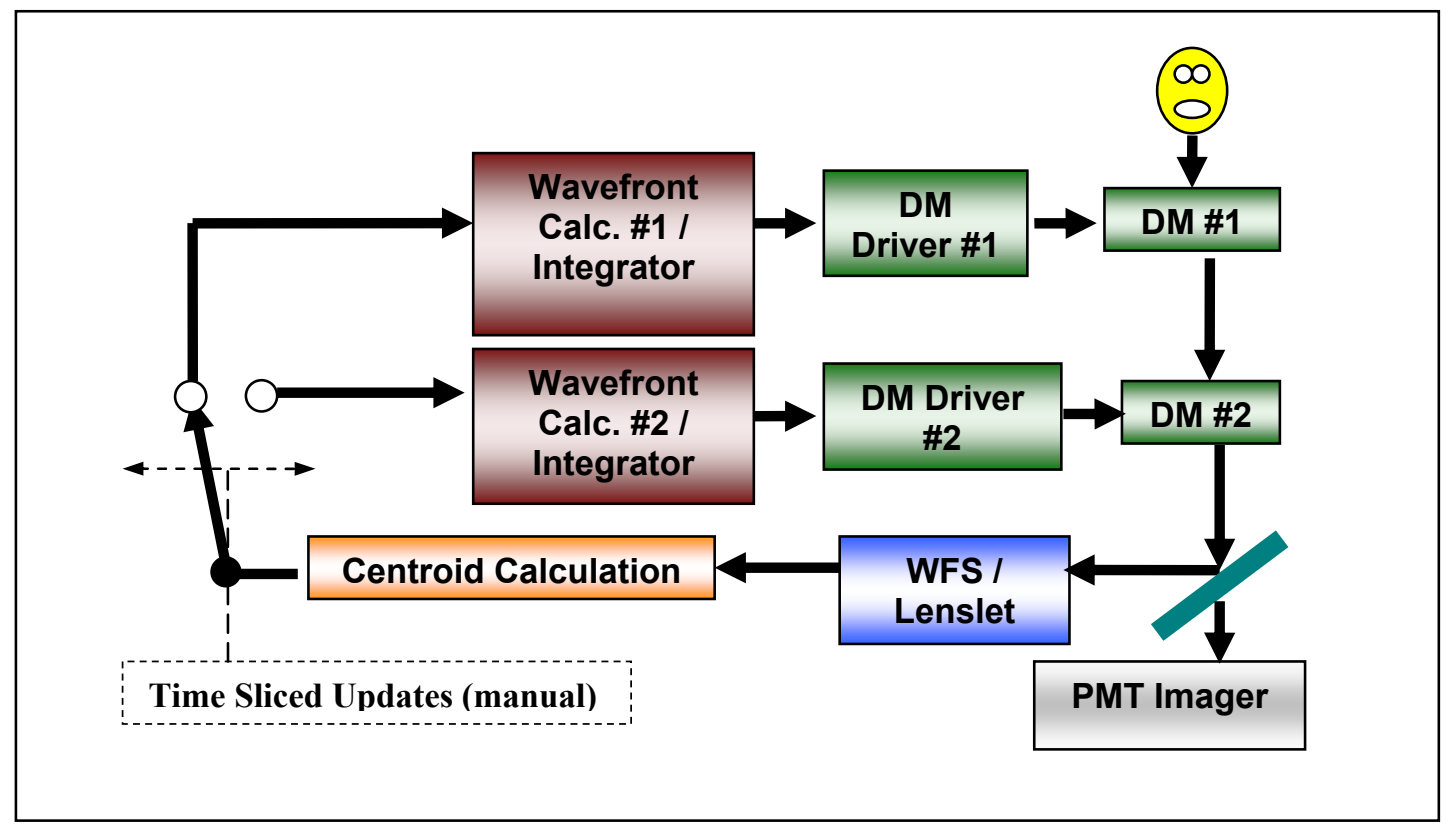

Figure 4 - Dual DM Operational Schematic: The Dual DM AO subsystem schematic is shown here. DM switching is currently controlled by the operator. Future enhancements will automate this operation, taking into account system usage and wavefront RMS.

\section{Wavefront Sensing and Correction}

The light source for the system is a low coherence Super Luminescent Diode (SLD) device, which is used both for the science imaging illumination and the Adaptive Optics beacon. The low coherence light source is preferred as high coherence sources are prone to speckle.

The wavefront sensor (SH-WFS) is a Shack-Hartmann configuration. The light path to the imaging device (SLO confocal imaging subsystem or OCT interference / spectrometer subsystem) is a common path with the SH-WFS, except for the final beam separation pellicle and the SH-WFS itself. The pellicle and SH-WFS are calibrated as a unit and a flat reference file is established, which corresponds to a flat wavefront in the imaging path, as seen at the pellicle. The SHWFS is built around a Dalsa Corporation 1M60 Pantera Camera and the Adaptive Optics Association (AOA) \#0500-30S-A lenslet. The SH-WFS camera is read by the AO computer at about a $20 \mathrm{~Hz}$ rate. With the optical and SH-WFS setup, the update rate is currently limited by the amount of light reflected from the eye.

As each frame is read from the camera, the zonal wavefront correction calculation is performed by the AO computer. The inverse of the calculated wavefront error is integrated with the previous error, using a gain of about 0.3 and loss of 0.98 , and the combined error is sent to the appropriate DM. Zonal operation is favored over Modal operation in these systems, using a control matrix that is calculated by measuring the actuator influence functions and then inverted into them into a control matrix. Modal operation is avoided, because calculations have shown that it would take a significant amount of time to calculate the necessary Zernike coefficients required to correct the wavefront using the full capabilities of the MEMS. These systems do not separate specific aberration types for control by one mirror or the other. The AOptix Bimorph mirror has 37 actuators, which provides some amount of high order correction, as well as the high stroke, low order corrections, however, the AOptix does not fit some error types, e.g., astigmatic errors, well enough to be made the only correction device for that error type. In this case the MEMS cooperates with the AOptix to provide optimal corrections. 
Since there is already an overlap between the two devices (e.g. for astigmatism), the decision was made to time slice the update of each DM. This time slice technique has the added benefit of eliminating any cross talk between the devices in a noisy situation.

\section{Woofer (AOptix Bimorph) Focus capability}

One of the major benefits of including a woofer type DM is its ability to provide retinal focusing capability for the system. The retina of the human eye is approximately $300 \mu \mathrm{m}$ thick and so it is necessary to focus at different depths to study specific structures in the eye. Figure \#5 is an overview of the retina.

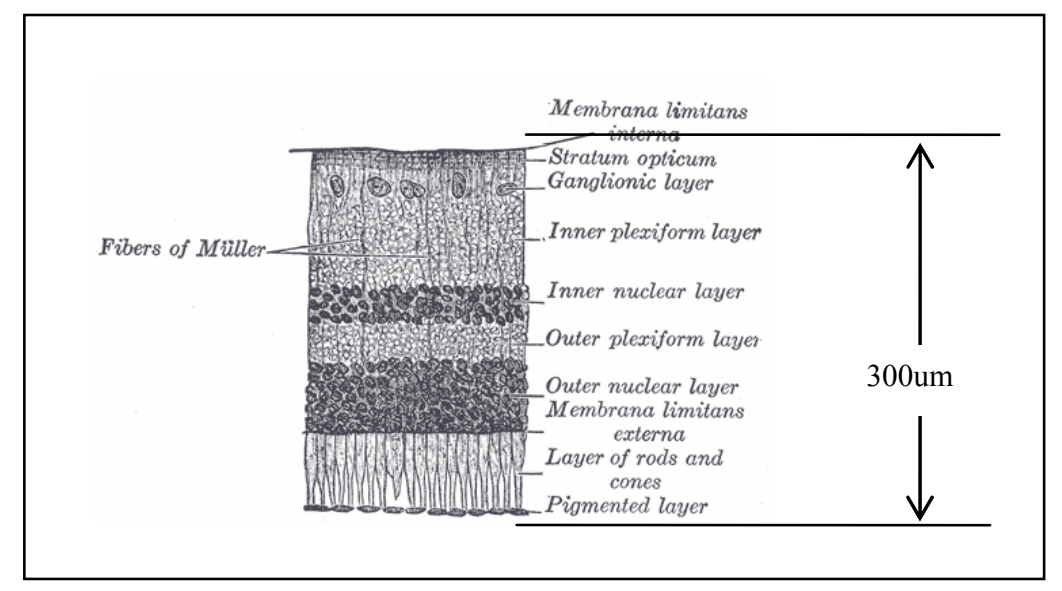

Figure 5 [21] - Thickness of Retina requires depth focusing: To allow the system to focus on the individual structures of the retina, the focus must be variable over 1 diopter of power for a normal eye. More power may be necessary in abnormal retinal structures.

Focusing in the retina is done by adding a centroid focus difference matrix, containing only the desired focus shift, to the wavefront sensor centroid readings. This combined error is then corrected by the AO system in closed loop by the woofer DM, thus introducing an artificial defocus. The use of a closed loop configuration avoids detailed calibration of the actuators of the DM, therefore changes in the system are easily handled using the normal calibration procedures. This method can be used to introduce any prescribed error that can be generated for the centroid matrix. This functionality is shown in Figure \#2. The user interface provides the ability to change the magnitude of the difference matrix over about a \pm 1.5 diopter range. The "flat" reference used by the wavefront sensor camera is unchanged during this period of time.

The woofer DM has a $10 \mathrm{~mm}$ aperture. The amount of stroke required to focus through the retina is calculated based on the change in power needed to move the focus from the front to the rear of the retina. The distance inside the human eye, from the lens to the front of the retina, is $\sim 17.8 \mathrm{~mm}$. This focusing distance for the lens system of the eye gives about $1 / 17.8 \mathrm{~mm}=56.2$ diopters. The thickness of a normal retina is approximately $300 \mu \mathrm{m}$, therefore the power required to focus at the rear of the eyeball is $1 / 18.1 \mathrm{~mm}=55.2$ diopters. Thus the change is about 1 diopter of power, which is about $12 \mu \mathrm{m}\left[\left(5 \mathrm{~mm}^{\wedge} 2\right) / 2\right]$ of optical stroke $(6 \mu \mathrm{m}$ mechanical). Thus we have used about $1 / 6$ of the available woofer stroke. Figure \#6 shows that the woofer DM provides an excellent solution to the retinal focus requirements, no additional cost to the system, and the woofer DM is at a pupil conjugate plane, therefore the focus can be changed without incurring the need to introduce an optical trombone, etc. This avoids shifting the pupil conjugate planes, which would degrade system performance. 


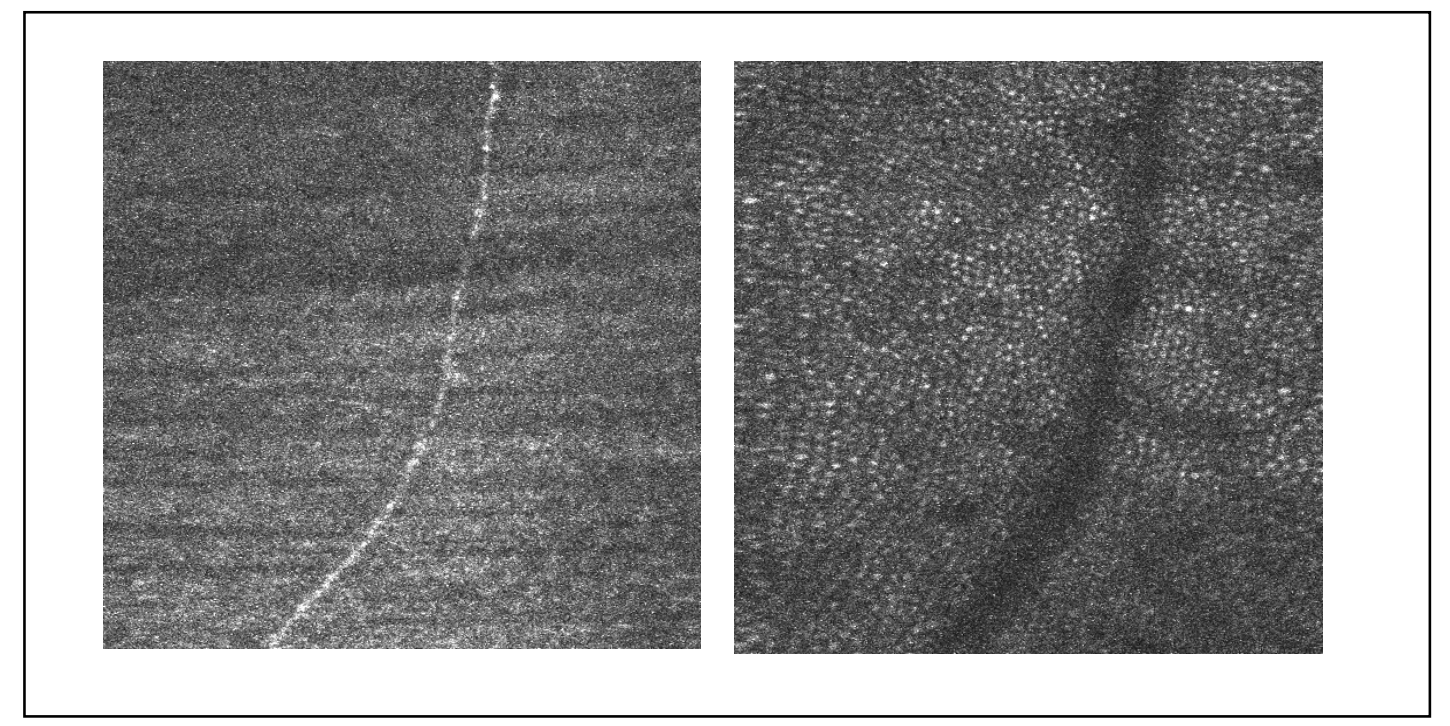

Figure 6 - Retinal focus capability using Dual DM (in-vivo human eye): These are single frame images extracted from an in-vivo video of the human retina. Both were taken at the same location but at different focus depths, using the defocus capability of the "woofer" DM. The left image is the nerve fiber layer and the right image shows the same area, but the photoreceptors are now evident. These images were taken at $\lambda \sim=840 \mathrm{~nm}$. Position: $3^{\circ} \mathrm{Nasal} / 3^{\circ}$ Superior, $1.1^{\circ} \times 1.1^{\circ}$

\section{Clinical Ease of Use}

The other facets of operation that are enhanced by the wide range correction capabilities of this system are the setup and increased size of the subject pool that is available to researchers, without resorting to trial lenses in the system. The elimination of trail lenses, except for coarse correction of spectacle type aberrations, is important for two reasons. First, the lenses introduce back reflections, which can be eliminated by tilting the lenses, which in turn introduces its own set of aberrations. A second reason for eliminating the trial lenses is that the AO-OCT system has to compensate, in the reference arm, for any additional optics that are used in the sample arm. The trial lens can be compensated for by adding optics to the reference arm, but that is time consuming and difficult. 


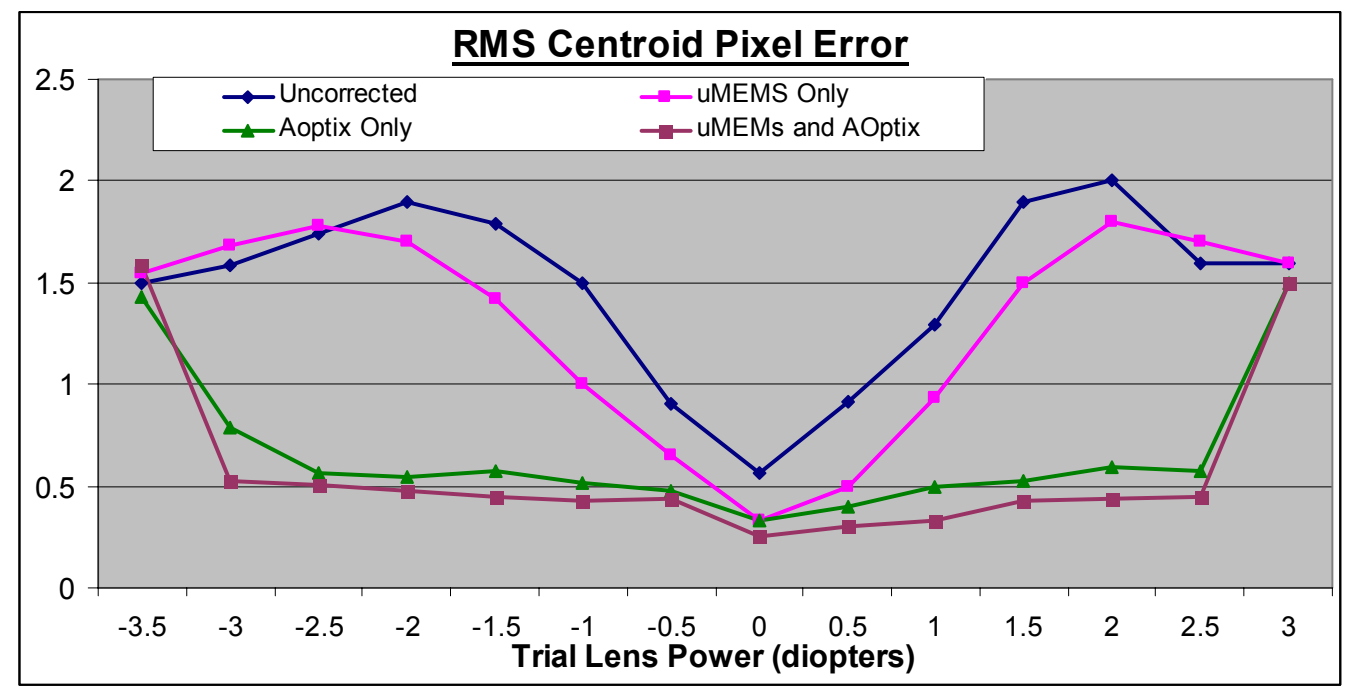

Figure 7 - Dual DM AO Dynamic Range :The "woofer" and "tweeter" DM configuration provides a range of about 5 diopters of power, which can be used for focusing through the retina and, additionally, easing the setup requirements for the subject. Data Provided by: Robert Zawadzki - UCDMC

The woofer DM widens the system range of correction from approximately 1.5 diopters, when using only the MEMS, to about 5 diopters, when using a combination of the woofer and tweeter DMs. A MEMS today typically is limited to approximately $4 \mu \mathrm{m}$ of optical stroke ( $2 \mu \mathrm{m}$ mechanical), as compared to the AOptix Bimorph, which has about $72 \mu \mathrm{m}$ of optical stroke [16]. If the system is optically aligned to center the useful stroke, then the subject pool can be increased to allow people having \pm 1.5 diopters of spectacle aberration. This takes into consideration the 1.0 diopter that will be used for retinal focusing and system calibration differences of about \pm 0.5 diopters. Figure $\# 7$, is a graph that shows the correction capability of the current AOptix Bimorph and MEMS combinations.

\section{RESULTS}

\section{AO Results when testing on a Mechanical Eye Correction}

The dual deformable mirror systems have been tested both on mechanical eyes and on human subjects. Figure \#8 shows the results of a typical correction sequence on a mechanical eye. There are three regions marked on the plot. On the wavefront RMS and Strehl plots, the left region, marked by a horizontal red bar, shows the system when neither DM is correcting. The middle region, marked by the yellow horizontal bar, shows the correction being done by the AOptix only. The final region, on the right and marked with a green horizontal line, shows the correction with both DMs correcting the wavefront.

Frames 21, 41 and 141 were chosen as representative frames for each of the three correction regions shown in figure \#8. Using these three frames, the Zernike values are calculated and the results plotted in the lower graph of Figure \#8. The red, yellow and green bar colors in the Zernike plot correspond to the same colors for the horizontal colored bars in the wavefront and Strehl plots. The wavefront RMS is reduced from about $330 \mathrm{~nm}$ to about $70 \mathrm{~nm}(\sim \lambda / 12)$. 


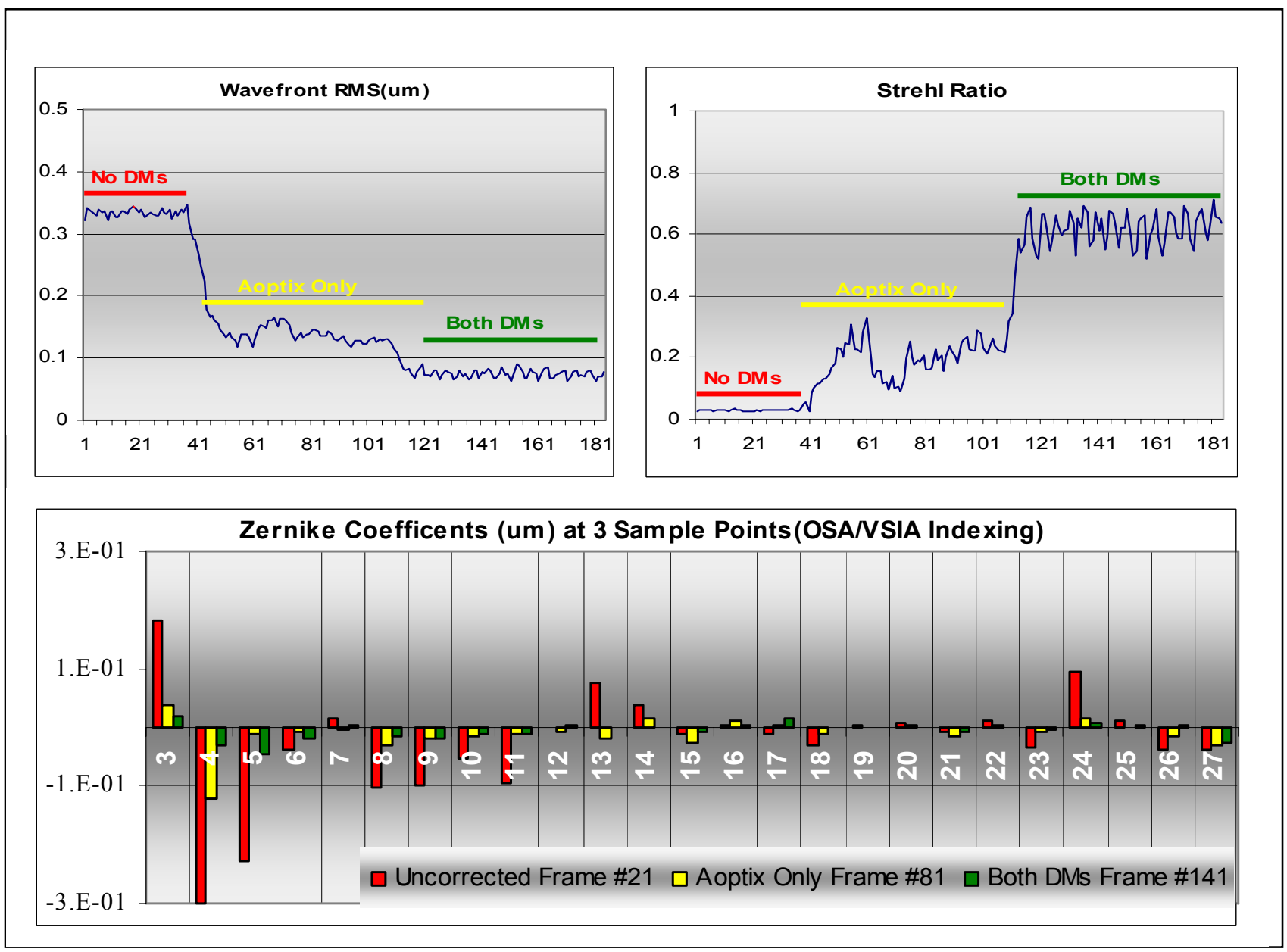

Figure 8 - Dual Deformable Mirror Operation (Mechanical Eye): These data shows the correction capabilities of the Dual DM system when used on a mechanical eye. The graphs show: 1) [red] the uncorrected by DMs, 2) [yellow] the "woofer" only correction and, 3) [green] both "woofer" and "tweeter" correction. RMS is reduced from about $330 \mathrm{~nm}$ to $70 \mathrm{~nm}$.

\section{AO Results when testing on a Human Eye}

Figure \#9 shows the same type as the mechanical eye above, but the data as collected on a human subject. The wavefront RMS and Strehl plots are similar to the plots shown in the mechanical eye plots in Figure \#8, except for the two blue, "manual defocus", regions on the plots. These "manual defocus" regions correspond to the times when the operator is changing the defocus position in the retina. After the changes are made, the wavefront RMS again shows the DMs correcting the introduced defocus error and the RMS value drops back down to about 70nm of RMS error at that point. T he Zernike coefficient plot in Figure \#9, corresponds to the red, yellow and green regions of the wavefront RMS graphs. These Zernike coefficients were calculated at three individual frames, selecting one from each region. 


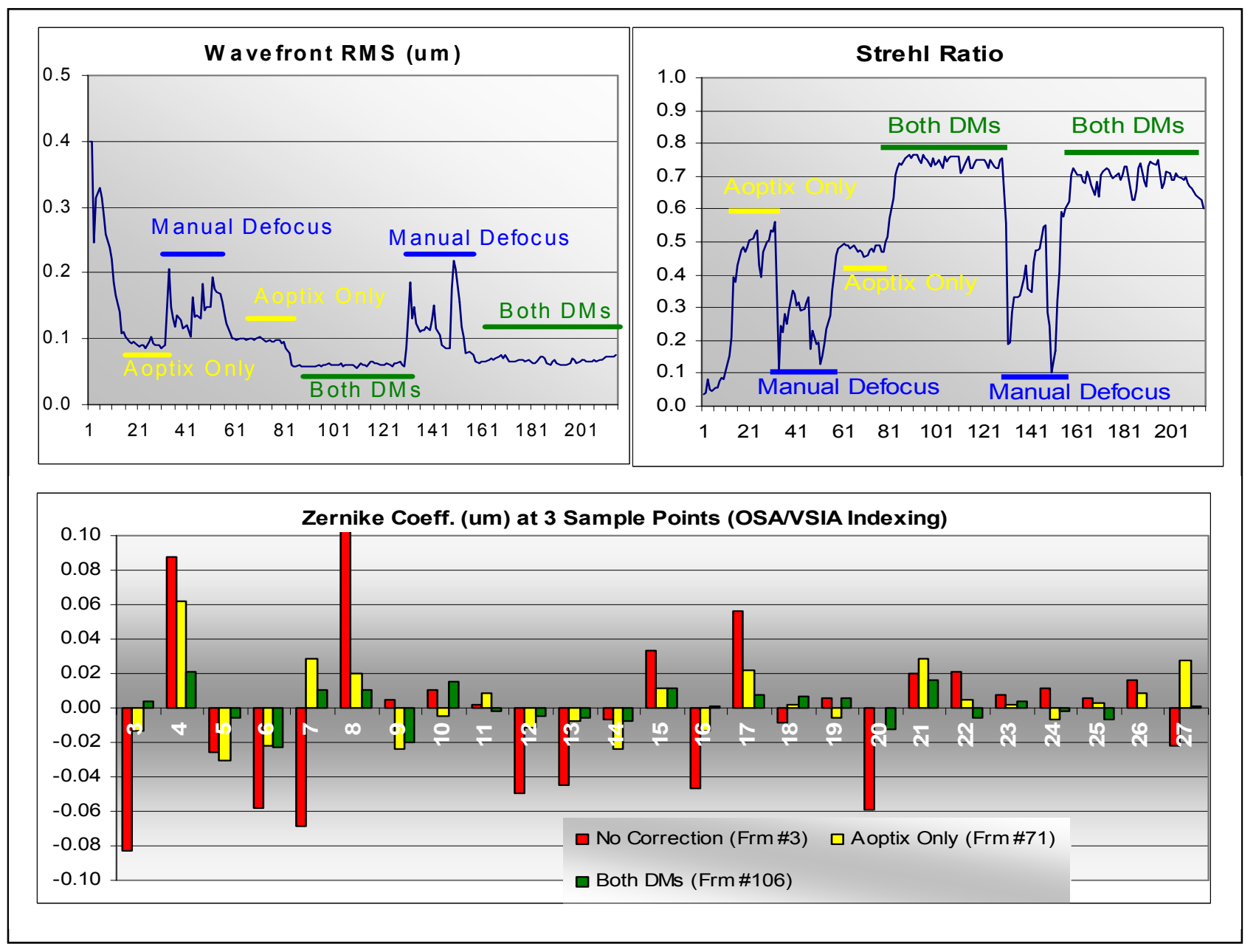

Figure 9 - Dual DM Operation on Human Eye (includes Manual Defocus): These data provides the wavefront correction results for a human eye. The graphs show: 1) [red] The uncorrected by DMs, 2) [yellow] The "woofer" only correction and, 3) [green] both "woofer" and "tweeter" correction. The violet colored bars are regions where the operator changed the focus position in the retina.

Figure \#10 shows images that demonstrate the uncorrected, correction using the woofer only, and the woofer-tweeter corrected images for a human subject. 


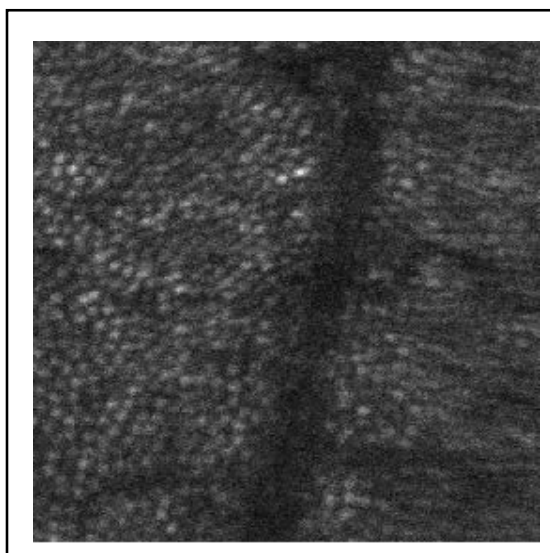

AO Off

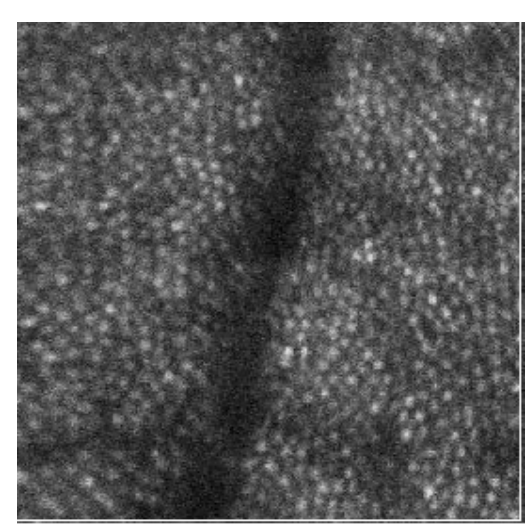

AOptix Only

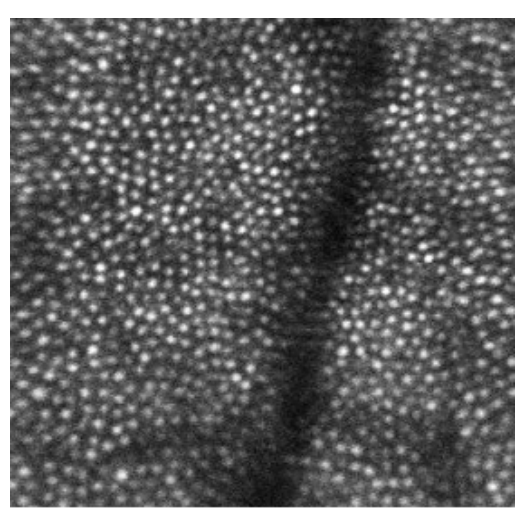

Both DMs

Figure 10 - Dual Deformable Mirror Operation (in-vivo human eye): These in-vivo images of the human retina were taken with an $840 \mathrm{~nm}$ SLD. The imaging area was at the retinal location of $3^{\circ}$ Nasal and $3^{\circ}$ Superior and the field of view is $1.1^{\circ}$.

\section{CONCLUSIONS}

We have shown that the dual deformable mirrors in the AO-SLO and the AO-OCT systems can compensate for relatively high amplitude, low order aberrations, and high order, low amplitude aberrations in the human eye. The invivo correction provides close to diffraction limited quality retinal images and the "woofer" deformable mirror allows focus capability through the retinal layers of the eye. The dual DMs reduce setup time and make available a significantly larger subject pool, due to the increased range of the AO correction in the system.

\section{ACKNOWLEDGEMENTS}

This research was supported by the NIH grant EY014365, NIH grant EY014743, and performed under the auspices of the U.S. Department of Energy by University of California, Lawrence Livermore National Laboratory under Contract W7405-Eng-48. All testing and imaging of subjects with this system was performed at the UC Davis Medical Center, Sacramento, CA., in collaboration with Dr. J.S. Werner's group, and at Doheny Eye Institute (USC Med Center), Los Angeles, CA., in collaboration with Dr. S. Sadda's group.

\section{REFERENCES}

1. R. Webb and G. Hughes, "Scanning laser ophthalmoscope," IEEE Trans. on Bio. Eng. 28, 488-492 (1981)

2. R. Webb, G. Hughes, and F. Delori, “Confocal scanning laser ophthalmoscope”, Appl. Opt. 26, 1492-1499 (1987)

3. Dreher, J. Bille, and R. Weinreb, "Active optical depth resolution improvement of the laser tomography scanner", Appl. Opt. 28, 804-808 (1989)

4. J. Liang, D. Williams, and D. Miller, "Supernormal vision and high-resolution retinal imaging through adaptive optics", J. Opt. Am. A 14, 2884-2892 (1997). 
5. Roorda, F. Romero-Borja, W. Donnelly, H. Queener, T. Herbert, and M. Campbell, “Adaptive optics scanning laser ophthalmoscopy", Opt. Exp. 10. 405-412 (2002).

6. Y. Zhang, S. Poonja, and A. Roorda, "Adaptive optics scanning laser ophthalmoscope using a micro-electromechanical (MEMS) deformable mirror," Proc. of SPIE 6138 (2006)

7. H. Hofer, L. Chen, G. Yoon, B. Singer, Y. Yamauchi, and D. Williams, "Improvement in retinal image quality with dynamic correction of the eye's aberration," Optics Express, 8, 631-643 (2001)

8. F. Vargas-Martin, P. Prieto, and P. Artal, "Correction of the aberrations in the human eye with a liquid-crystal spatial light modulator: limits to performance", J. Opt. Am. A 15, 2552-2562 (1998).

9. T. Bifano, J. Perreault, P. Bierden, and C. Dimas, "Micromachined deformable mirrors for adaptive optics," Proc. of SPIE 4825, 10-13 (2002)

10. N. Doble and D. Williams, "The applications of MEMS technology for adaptive optics in vision science," IEEE Quantum Electronics, 10, 629-635 (2004)

11. J. Porter, A. Guirao, I. Cox and D. Williams, "Monochromatic aberrations of the human eye in a large populations," J. Opt. Am. A. 18, 1793-1803, (2001)

12. L. Thibos, X. Hong, A. Bradley and X. Cheng, "Statistical variation of aberration structure and image quality in a normal population of healthy eyes," J. Opt. Am. A. 19, 2329-2348 (2002)

13. Elsner, "Fundamental properties of the retina", 205-234, "Adaptive Optics for Vision Science", Wiley\& Sons

14. D. Horsley, H. Park, S. Laut and J. Werner, "Characterization for vision science applications of a bimorph deformable mirror using phase-shifting interferometry", SPIE 5688, 133-144 (2005)

15. D.T. Miller, J. Qu, R.S. Jonnal, and K. Thorn, "Coherence gating and adaptive optics in the eye," in Coherence Domain Optical Methods and Optical Coherence Tomography in Biomedicine VII, V.V. Tuchin, J.A. Izatt, J.G. Fujimoto, eds., Proc. SPIE 4956, 65-72 (2003).

16. B. Hermann, E.J. Fernandez, A. Unterhubner, H. Sattmann, A.F. Fercher, and W. Drexler, P.M. Prieto and P. Artal, "Adaptive-optics ultrahigh-resolution optical coherence tomography," Opt. Lett. 29, 2142-2144 (2004).

17. Y. Zhang, J. Rha, R.S. Jonnal, D.T. Miller, "Adaptive optics parallel spectral domain optical coherence tomography for imaging the living retina," Opt. Express 13, 4792-4811 (2005), http://www.opticsexpress.org/abstract.cfm?URI=OPEX-13-12-4792.

18. E. J. Fernández, B. Považay, B. Hermann, A. Unterhuber, H. Sattmann, P.M. Prieto, R. Leitgeb, P. Ahnelt, P. Artal and W. Drexler, "Three-dimensional adaptive optics ultrahigh-resolution optical coherence tomography using a liquid crystal spatial light modulator " Vision Research 45, 3432-3444 (2005)

19. R.J. Zawadzki, S. Jones, S.S. Olivier, M. Zhao, B.A. Bower, J.A. Izatt, S.S. Choi, S. Laut, and J.S. Werner, "Adaptive-optics optical coherence tomography for high-resolution and high-speed 3D retinal in vivo imaging," Opt. Express 13, 8532-8546 (2005), http://www.opticsinfobase.org/abstract.cfm?URI=oe-13-21-8532

20. L.N. Thibos, R.A. Applegate, J.T. Schwiegerling, and R. Web, "Standards for reporting optical aberrations of eyes," J. Refract. Surg. 18, 652-660 (2000)

21. Plate from Online Edition of "Gray's Anatomy of the Human Body", 20th U.S. edition (1918). 